\title{
Recovering differential pencils with spectral boundary conditions and spectral jump conditions
}

\author{
Yasser Khalilii* and Dumitru Baleanu
}

\section{"Correspondence:}

y.khalili@sanru.ac.ir

'Department of Basic Sciences, Sari

Agricultural Sciences and Natural

Resources University, 578, Sari, Iran

Full list of author information is

available at the end of the article

\section{Springer}

\begin{abstract}
In this work, we discuss the inverse problem for second order differential pencils with boundary and jump conditions dependent on the spectral parameter. We establish the following uniqueness theorems: $(i)$ the potentials $q_{k}(x)$ and boundary conditions of such a problem can be uniquely established by some information on eigenfunctions at some internal point $b \in\left(\frac{\pi}{2}, \pi\right)$ and parts of two spectra; (ii) if one boundary condition and the potentials $a_{k}(x)$ are prescribed on the interval $[\pi / 2(1-\alpha), \pi]$ for some $\alpha \in(0,1)$, then parts of spectra $S \subseteq \sigma(L)$ are enough to determine the potentials $q_{k}(x)$ on the whole interval $[0, \pi]$ and another boundary condition.
\end{abstract}

MSC: 34A36; 65L09; 34L05

Keywords: Inverse problem; Differential pencil; Spectral boundary condition; Spectral jump condition

\section{Introduction}

Inverse spectral problems are a branch of science that seeks to determine the coefficients of the boundary value problems from their spectral characteristics. This kind of problems often arise in mathematics, mechanics, physics, electronics, geophysics, and various branches of natural sciences and engineering [2, 3, 14, 17, 18, 24]. First studies and results of inverse problems for classical Sturm-Liouville operators were given by Ambartsumyan in 1929 [1], and this field of science has been developed by many researchers in the next years $[8,21,29,33,37,38]$. In particular, some aspects of the inverse problem theory for differential pencils and for spectral jump and boundary conditions have been investigated in $[4,6,23,29-35]$.

In this paper, we investigate the boundary value problem $L:=L\left(q_{1}, q_{0}, h_{1}, h_{0}, H_{1}, H_{0}, \alpha\right.$, $\beta, \gamma)$ for the differential pencil

$$
-y^{\prime \prime}+\left(2 \rho q_{1}(x)+q_{0}(x)\right) y=\rho^{2} y, \quad x \in(0, \pi),
$$

(c) The Author(s) 2020. This article is licensed under a Creative Commons Attribution 4.0 International License, which permits use, sharing, adaptation, distribution and reproduction in any medium or format, as long as you give appropriate credit to the original author(s) and the source, provide a link to the Creative Commons licence, and indicate if changes were made. The images or other third party material in this article are included in the article's Creative Commons licence, unless indicated otherwise in a credit line to the material. If material is not included in the article's Creative Commons licence and your intended use is not permitted by statutory regulation or exceeds the permitted use, you will need to obtain permission directly from the copyright holder. To view a copy of this licence, visit http://creativecommons.org/licenses/by/4.0/. 
with boundary conditions

$$
\begin{aligned}
& U(y):=y^{\prime}(0)-\left(h_{1} \rho+h_{0}\right) y(0)=0, \\
& V(y):=y^{\prime}(\pi)+\left(H_{1} \rho+H_{0}\right) y(\pi)=0,
\end{aligned}
$$

and the interior discontinuity

$$
\begin{aligned}
& y\left(\frac{\pi}{2}+0, \rho\right)=\alpha y\left(\frac{\pi}{2}-0, \rho\right), \\
& y^{\prime}\left(\frac{\pi}{2}+0, \rho\right)=\alpha^{-1} y^{\prime}\left(\frac{\pi}{2}-0, \rho\right)+(\beta \rho+\gamma) y\left(\frac{\pi}{2}-0, \rho\right) .
\end{aligned}
$$

The parameters $h_{k}, H_{k}, k=0,1\left(h_{1}, H_{1} \neq \pm i\right), \alpha>0, \beta$ and $\gamma$ are complex, and $\rho$ is a spectral parameter. The complex-valued functions $q_{k}(x)$ belong to the space $W_{2}^{k}(0, \pi)$.

Some special cases of problem (1.1)-(1.4) arise after an application of the method of the separation of variables to the varied assortment of physical problems. For example, some of the problems with boundary conditions depending on the spectral parameter occur in the theory of small vibrations of a damped string and freezing of the liquid [19, 26]. These problems also appear in the connection with an acoustic wave propagation in a rectangular duct with a uniform mean flow profile and walls with finite acoustic impedance [13]. Moreover, boundary value problems with discontinuity conditions arise in heat and mass transfer problems [16], in vibrating string problems when the string is loaded additionally with point masses [26], and in diffraction problems [27].

To recover the potential on all interval and all coefficients in boundary conditions in the usual case, it is necessary to know two spectra of the boundary value problem with different boundary conditions [36]. Indeed, if a finite number of eigenvalues are deleted, the potential is not uniquely determined by one full spectrum and one partial spectrum. Mochizuki and Trooshin [20] showed that the spectral data of parts of two spectra and a set of values of eigenfunctions at some internal point suffice to determine the potential, and they addressed the interior inverse problem of Sturm-Liouville operators on the finite interval $[0,1]$. Afterwards, this technique has been used by some authors to survey the inverse problem of Sturm-Liouville operators in various forms [10, 22, 25, 29, 33]. Alongside this method, in [9], Hochstadt and Lieberman found the half inverse problem method and showed that if the potential is prescribed on $[1 / 2,1]$, one spectrum can uniquely determine the potential on the whole interval $[0,1]$. Hochstadt-Lieberman type theorem was also investigated by many scholars for differential operators in the next years $[4,11,12,30,31]$. Then Gesztesy and Simon generalized Hochstadt-Lieberman type theorem and recovered the Sturm-Liouville operator with Robin boundary conditions from parts of one spectrum and partial information on the potential [7]. They showed that if the coefficient $h_{0}$ and the potential are provided on $[0,1 / 2+\alpha / 2]$ for some $\alpha \in(0,1)$, then parts of one spectrum can give the coefficient $h_{1}$ and the potential on all of $[0,1]$. Insofar as we know, Mochizuki-Trooshin and Hochstadt-Lieberman type theorems for differential pencils with spectral boundary and jump conditions have not been considered before. The target of this work is to investigate two inverse problems to $L$ by these two techniques. We would like to investigate the inverse problems for $L$ by some information on eigenfunctions at some internal point and parts of two spectra taking Mochizuki-Trooshin type 
theorem as well as from partial information on the potentials and parts of a finite number of spectra by Gesztesy-Simon type theorem. This brings certain difficulties, and the results obtained are a generalization of the classical Sturm-Liouville problems.

The main goal of this paper is to present the potentials $q_{k}(x)$ and the parameters $h_{k}, H_{k}, k=0,1$ by developing the ideas of the Mochizuki-Trooshin and Gesztesy-Simon methods $[7,20]$. The present paper is organized as follows. Section 2 is devoted to some preliminaries. In Sect. 3, two uniqueness theorems for boundary value problem (1.1)-(1.4) are proved.

\section{Preliminaries}

In the first part of the paper, we provide the solution of the boundary value problem $L$ and its spectral characteristics. At first, we remind the following notation from [5].

The values of the parameter $\lambda=\rho^{2}$ for which $L$ has nonzero solutions are called eigenvalues, and the corresponding nontrivial solutions are called eigenfunctions. The set of eigenvalues is called the spectrum of $L$.

Let $y(x, \rho)$ be the solution of differential pencil (1.1) under the initial conditions $y(0, \rho)=$ $1, y^{\prime}(0, \rho)=h_{1} \rho+h_{0}$ and jump conditions (1.4). From [23,34], this solution can be obtained in the following form for sufficiently large $\rho$ :

$$
\begin{aligned}
y(x, \rho)= & \cos (\rho x-Q(x))+h_{1} \sin (\rho x-Q(x))+O\left(\frac{1}{\rho} \exp (|\Im \rho| x)\right), \quad x<\frac{\pi}{2}, \\
y(x, \rho)= & \left(\alpha^{+}-\frac{1}{2} \beta h_{1}\right)\left(\cos (\rho x-Q(x))+A_{1} \sin (\rho x-Q(x))\right) \\
& +\left(\alpha^{-}+\frac{1}{2} \beta h_{1}\right)(\cos (\rho(\pi-x)-Q(\pi)+Q(x)) \\
& \left.+A_{2} \sin (\rho(\pi-x)-Q(\pi)+Q(x))\right) \\
& +O\left(\frac{1}{\rho} \exp (|\Im \rho| x)\right), \quad x>\frac{\pi}{2},
\end{aligned}
$$

where $Q(x)=\int_{0}^{x} q_{1}(t) d t, A_{1}=\frac{2 \alpha^{+} h_{1}+\beta}{2 \alpha^{+}-\beta h_{1}}$, and $A_{2}=\frac{2 \alpha^{-} h_{1}-\beta}{2 \alpha^{-}+\beta h_{1}}$ in which $\alpha^{ \pm}=\frac{1}{2}\left(\alpha \pm \alpha^{-1}\right)$. Since

$$
\frac{1}{\sqrt{1+h_{1}^{2}}}=\cos \left(\frac{1}{2 i} \ln \frac{i-h_{1}}{i+h_{1}}\right), \quad \frac{h_{1}}{\sqrt{1+h_{1}^{2}}}=\sin \left(\frac{1}{2 i} \ln \frac{i-h_{1}}{i+h_{1}}\right)
$$

and analogously

$$
\frac{1}{\sqrt{1+A_{j}^{2}}}=\cos \left(\frac{1}{2 i} \ln \frac{i-A_{j}}{i+A_{j}}\right), \quad \frac{A_{j}}{\sqrt{1+A_{j}^{2}}}=\sin \left(\frac{1}{2 i} \ln \frac{i-A_{j}}{i+A_{j}}\right), \quad j=1,2,
$$

we have formulae (2.1) and (2.2) as follows for sufficiently large $\rho$ :

$$
\begin{aligned}
y(x, \rho)= & \sqrt{1+h_{1}^{2}} \cos \left(\frac{1}{2 i} \ln \frac{i-h_{1}}{i+h_{1}}-(\rho x-Q(x))\right) \\
& +O\left(\frac{1}{\rho} \exp (|\Im \rho| x)\right), \quad x<\frac{\pi}{2}, \\
y(x, \rho)= & \left(\alpha^{+}-\frac{1}{2} \beta h_{1}\right) \sqrt{1+A_{1}^{2}} \cos \left(\frac{1}{2 i} \ln \frac{i-A_{1}}{i+A_{1}}-(\rho x-Q(x))\right)
\end{aligned}
$$




$$
\begin{aligned}
& +\left(\alpha^{-}+\frac{1}{2} \beta h_{1}\right) \sqrt{1+A_{2}^{2}} \cos \left(\frac{1}{2 i} \ln \frac{i-A_{2}}{i+A_{2}}-(\rho(\pi-x)-Q(\pi)+Q(x))\right) \\
& +O\left(\frac{1}{\rho} \exp (|\Im \rho| x)\right), \quad x>\frac{\pi}{2} .
\end{aligned}
$$

Moreover, we know that these functions and derivatives with respect to $x$ are entire in $\rho$ of exponential type.

We denote by $\Delta(\rho):=V(y(x, \rho))$ the characteristic function for $L$. This relation together with (1.3) and (2.4) gives for sufficiently large $\rho$ :

$$
\begin{aligned}
\Delta(\rho)= & \rho \sqrt{1+H_{1}^{2}}\left(\left(\alpha^{+}-\frac{1}{2} \beta h_{1}\right)\right. \\
& \times \sqrt{1+A_{1}^{2}} \sin \left(\frac{1}{2 i} \ln \frac{i-H_{1}}{i+H_{1}}+\frac{1}{2 i} \ln \frac{i-A_{1}}{i+A_{1}}-(\rho \pi-Q(\pi))\right) \\
& \left.+\left(\alpha^{-}+\frac{1}{2} \beta h_{1}\right) \sqrt{1+A_{2}^{2}} \sin \left(\frac{1}{2 i} \ln \frac{i-H_{1}}{i+H_{1}}+\frac{1}{2 i} \ln \frac{i-A_{2}}{i+A_{2}}\right)\right) \\
& +O(\exp (|\Im \rho| \pi)) .
\end{aligned}
$$

The roots of this characteristic function are the eigenvalues of $L$ [5], and these eigenvalues have the following asymptotic formula for sufficiently large $n$ :

$$
\rho_{n}=n+\omega+O\left(\frac{1}{n}\right)
$$

where $\omega=\frac{1}{\pi} Q(\pi)+\frac{1}{2 \pi i} \ln \frac{i-H_{1}}{i+H_{1}}+\frac{1}{2 \pi i} \ln \frac{i-A_{1}}{i+A_{1}}$. Also, using the known method [5], one gets

$$
|\Delta(\rho)| \geq C_{\delta}|\rho| \exp (|\Im \rho| \pi)
$$

for large enough $\rho \in G_{\delta}:=\left\{\rho \in \mathbb{C} ;\left|\rho-\rho_{n}\right| \geq \delta, \forall n,\right\}$ and some positive constant $C_{\delta}$.

In virtue of Ref. [7, 15], we bring the following lemma which is momentous to demonstrating the main results of our article.

Lemma 2.1 For any entire function $g(\rho) \neq 0$ of exponential type, the following inequality holds:

$$
\lim _{r \rightarrow \infty} \frac{n(r)}{r} \leq \frac{1}{2 \pi} \int_{0}^{2 \pi} h_{g}(\theta) d \theta
$$

where $n(r)$ is the number of zeros of $g(\rho)$ in the disk $|\rho| \leq r$ and $h_{g}(\theta):=\lim \sup _{r \rightarrow \infty} \frac{\ln \left|g\left(r e^{i \theta}\right)\right|}{r}$ with $\rho=r e^{i \theta}$.

Lemma 2.2 Assume that $f(z)$ is an entire function of order less than one. If $\lim _{|x| \rightarrow \infty, x \in \mathbb{R}} f(i x)=0$, then $f(z)=0$ on the whole complex plane.

\section{Inverse problem with partial information}

In this section, two uniqueness theorems are brought that are the main results of this work. We would like to recover the differential pencil and the coefficients used in 
boundary conditions from some information on eigenfunctions at some interior point and parts of two spectra in Theorem 3.1. Taking partial information on the potentials and a subset of eigenvalues, we also study this inverse problem in Theorem 3.3. So, we consider a boundary value problem $\widetilde{L}:=L\left(\widetilde{q}_{1}, \widetilde{q}_{0}, \widetilde{h}_{1}, \widetilde{h}_{0}, \widetilde{H}_{1}, \widetilde{H}_{0}, \alpha, \beta, \gamma\right)$ beside $L:=L\left(q_{1}, q_{0}, h_{1}, h_{0}, H_{1}, H_{0}, \alpha, \beta, \gamma\right)$. We note that the parameters $\alpha, \beta$, and $\gamma$ are known a priori. Also, if a symbol shows an object in $L$, then the same symbol with tilde shows the corresponding object in $\widetilde{L}$.

Let $l(n)$ and $r(n)$ be two sequences of the natural numbers such that

$$
\begin{array}{ll}
l(n)=\frac{n}{\sigma_{1}}\left(1+\epsilon_{1 n}\right), & 0<\sigma_{1} \leq 1, \epsilon_{1 n} \longrightarrow 0, \\
r(n)=\frac{n}{\sigma_{2}}\left(1+\epsilon_{2 n}\right), & 0<\sigma_{2} \leq 1, \epsilon_{2 n} \longrightarrow 0,
\end{array}
$$

and let $\mu_{n}$ be the eigenvalues of $L_{1}:=L\left(q_{0}, q_{1}, h_{0}, h_{1}, \mathcal{H}_{0}, \mathcal{H}_{1}, \alpha, \beta, \gamma\right), \mathcal{H}_{k} \neq H_{k}, \mathcal{H}_{k} \in \mathbb{R}$ for $k=0,1$.

Theorem 3.1 Consider two sequences $l(n)$ and $r(n)$ such that $\sigma_{1}>\frac{2 b}{\pi}-1$ and $\sigma_{2}>2-\frac{2 b}{\pi}$ as $b \in\left(\frac{\pi}{2}, \pi\right)$. If, for any $n$,

$$
\begin{aligned}
& \lambda_{n}=\tilde{\lambda}_{n}, \quad \mu_{l(n)}=\tilde{\mu}_{l(n)}, \\
& \left\langle y_{r(n)}, \tilde{y}_{r(n)}\right\rangle_{x=b}=0,
\end{aligned}
$$

where $\langle y, z\rangle:=y z^{\prime}-y^{\prime} z$, then $q_{k}(x)=\widetilde{q}_{k}(x)$ a.e. on $[0, \pi]$ and $h_{k}=\widetilde{h}_{k}, H_{k}=\widetilde{H}_{k}, k=0,1$.

It is needed to express the following lemma to prove Theorem 3.1.

Lemma 3.2 Consider a sequence of natural numbers

$$
m(n)=\frac{n}{\sigma}\left(1+\epsilon_{n}\right), \quad 0<\sigma \leq 1, \epsilon_{n} \longrightarrow 0
$$

(1) Let $\sigma>\frac{2 b}{\pi}$ for $b \in\left(0, \frac{\pi}{2}\right)$.If, for any $n$,

$$
\lambda_{m(n)}=\tilde{\lambda}_{m(n)}, \quad\left\langle y_{m(n)}, \tilde{y}_{m(n)}\right\rangle_{x=b}=0,
$$

then $q_{k}(x)=\widetilde{q}_{k}(x)$ a.e. on $[0, b]$ and $h_{k}=\widetilde{h}_{k}, k=0,1$.

(2) Let $\sigma>2-\frac{2 b}{\pi}$ for $b \in\left(\frac{\pi}{2}, \pi\right)$. If, for any $n$,

$$
\lambda_{m(n)}=\tilde{\lambda}_{m(n)}, \quad\left\langle y_{m(n)}, \tilde{y}_{m(n)}\right\rangle_{x=b}=0,
$$

then $q_{k}(x)=\widetilde{q}_{k}(x)$ a.e. on $[b, \pi]$ and $H_{k}=\widetilde{H}_{k}, k=0,1$.

Proof Let $y(x, \rho)$ be the solution to equation (1.1) with the initial conditions $y(0, \rho)=1$ and $y^{\prime}(0, \rho)=h_{1} \rho+h_{0}$. Also, let $\widetilde{y}(x, \rho)$ be the solution of the corresponding equation with tilde and with the initial conditions $\widetilde{y}(0, \rho)=1$ and $\widetilde{y}^{\prime}(0, \rho)=\widetilde{h}_{1} \rho+\widetilde{h}_{0}$. Multiplying (1.1) by 
$\tilde{y}(x, \rho)$ and the corresponding equation by $y(x, \rho)$, using the difference of these results, and integrating on $[0, b]$, we get

$$
\begin{aligned}
G_{b}(\rho) & :=\int_{0}^{b}\left(2 \rho Q_{1}(x)+Q_{0}(x)\right) y(x) \widetilde{y}(x) d x+\left(h_{1}-\widetilde{h}_{1}\right) \rho+h_{0}-\widetilde{h}_{0} \\
& =y^{\prime}(b) \widetilde{y}(b)-y(b) \widetilde{y}^{\prime}(b),
\end{aligned}
$$

where $Q_{k}(x)=q_{k}(x)-\widetilde{q}_{k}(x), k=0,1$. According to the assumptions of the theorem, we have

$$
G_{b}\left(\rho_{m(n)}\right)=0 .
$$

In the following, we must show that $G_{b}(\rho)=0$ for all $\rho \neq \rho_{n}$.

We hold the following integral representation for two bounded functions $H_{c}(x, t)$ and $H_{s}(x, t)$ :

$$
\begin{aligned}
y(x, \rho)= & \cos (\rho x-Q(x))+h_{1} \sin (\rho x-Q(x)) \\
& +\int_{0}^{x} H_{c}(x, t) \cos \rho t d t+\int_{0}^{x} H_{s}(x, t) \sin \rho t d t, \quad x<\frac{\pi}{2} .
\end{aligned}
$$

[34]. Thus, consider $Q_{ \pm}(x)=Q(x) \pm \widetilde{Q}(x)$,

$$
\begin{aligned}
y(x, \rho) \widetilde{y}(x, \rho)= & \frac{1+h_{1} \widetilde{h}_{1}}{2} \cos Q_{-}(x)+\frac{h_{1}-\widetilde{h}_{1}}{2} \sin Q_{-}(x) \\
& +\frac{1-h_{1} \widetilde{h}_{1}}{2} \cos 2\left(\rho x-Q_{+}(x)\right)-\frac{h_{1}+\widetilde{h}_{1}}{2} \sin 2\left(\rho x-Q_{+}(x)\right) \\
& +\int_{0}^{x} H_{c}^{\prime}(x, t) \cos \left(2 \rho t-Q_{+}(t)\right) d t \\
& +\int_{0}^{x} H_{s}^{\prime}(x, t) \sin \left(2 \rho t-Q_{+}(t)\right) d t, \quad x<\frac{\pi}{2}
\end{aligned}
$$

where $H_{c}^{\prime}(x, t)$ and $H_{s}^{\prime}(x, t)$ are bounded functions. Now, by taking (3.3), one gets

$$
|y(x, \rho) \widetilde{y}(x, \rho)| \leq M \exp (2|\Im \rho| x),
$$

and therefore this result together with (3.1) implies that

$$
\left|G_{b}(\rho)\right| \leq\left(M_{1}+M_{2}|\rho|\right) \exp (2 b r|\sin \theta|)
$$

Using the above result and considering the indicator

$$
h(\theta):=\limsup _{r \rightarrow \infty} \frac{\ln \left|G_{b}(r \exp (i \theta))\right|}{r},
$$

we obtain

$$
h(\theta) \leq 2 b|\sin \theta|
$$


and so

$$
\frac{1}{2 \pi} \int_{0}^{2 \pi} h(\theta) d \theta \leq \frac{b}{\pi} \int_{0}^{2 \pi}|\sin \theta| d \theta=\frac{4 b}{\pi} .
$$

Taking the hypothesis of the lemma and (2.5), one gets, for sufficiently large $r$,

$$
n(r) \geq 2 \sum_{\frac{n}{\sigma}\left(1+\frac{\omega}{n}+O\left(n^{-2}\right)\right)<r} 1=2 r \sigma[1+\epsilon(r)]
$$

in which $n(r)$ is the number of roots of $G_{b}(\rho)$ in the disk $|\rho| \leq r$. Thus, for $\sigma>\frac{2 b}{\pi}$, we obtain that

$$
\lim _{r \rightarrow \infty} \frac{n(r)}{r} \geq 2 \sigma>\frac{1}{2 \pi} \int_{0}^{2 \pi} h(\theta) d \theta
$$

Lemma 2.1 together with (3.8) yields that $G_{b}(\rho)=0$ on the whole complex plane.

Now, substituting (3.3) into (3.1), we can write that

$$
\begin{aligned}
& \left(h_{1}-\widetilde{h}_{1}\right) \rho+h_{0}-\widetilde{h}_{0} \\
& +\int_{0}^{b}\left(2 \rho Q_{1}(x)+Q_{0}(x)\right)\left[\frac{1+h_{1} \widetilde{h}_{1}}{2} \cos Q_{-}(x)+\frac{h_{1}-\widetilde{h}_{1}}{2} \sin Q_{-}(x)\right. \\
& \left.+\frac{1-h_{1} \widetilde{h}_{1}}{2} \cos 2\left(\rho x-Q_{+}(x)\right)-\frac{h_{1}+\widetilde{h}_{1}}{2} \sin 2\left(\rho x-Q_{+}(x)\right)\right] d x \\
& +\int_{0}^{b}\left(2 \rho Q_{1}(x)+Q_{0}(x)\right)\left[\int_{0}^{x} H_{c}^{\prime}(x, t) \cos \left(2 \rho t-Q_{+}(t)\right) d t\right. \\
& \left.+\int_{0}^{x} H_{s}^{\prime}(x, t) \sin \left(2 \rho t-Q_{+}(t)\right) d t\right] d x=0 .
\end{aligned}
$$

This can be rewritten as

$$
\begin{aligned}
& \left(h_{1}-\widetilde{h}_{1}\right) \rho+2 \rho \int_{0}^{b} Q_{1}(x)\left[\frac{1+h_{1} \widetilde{h}_{1}}{2} \cos Q_{-}(x)+\frac{h_{1}-\widetilde{h}_{1}}{2} \sin Q_{-}(x)\right] d x \\
& +h_{0}-\widetilde{h}_{0}+\int_{0}^{b} Q_{0}(x)\left[\frac{1+h_{1} \widetilde{h}_{1}}{2} \cos Q_{-}(x)+\frac{h_{1}-\widetilde{h}_{1}}{2} \sin Q_{-}(x)\right] d x \\
& +2 \rho \int_{0}^{b} \cos \left(2 \rho t-Q_{+}(t)\right)\left[\frac{1-h_{1} \widetilde{h}_{1}}{2} Q_{1}(t)+\int_{t}^{b} H_{c}^{\prime}(x, t) Q_{1}(x) d x\right] d t \\
& +2 \rho \int_{0}^{b} \sin \left(2 \rho t-Q_{+}(t)\right)\left[-\frac{h_{1}+\widetilde{h}_{1}}{2} Q_{1}(t)+\int_{t}^{b} H_{s}^{\prime}(x, t) Q_{1}(x) d x\right] d t \\
& +\int_{0}^{b} \cos \left(2 \rho t-Q_{+}(t)\right)\left[\frac{1-h_{1} \widetilde{h}_{1}}{2} Q_{0}(t)+\int_{t}^{b} H_{c}^{\prime}(x, t) Q_{0}(x) d x\right] d t \\
& +\int_{0}^{b} \sin \left(2 \rho t-Q_{+}(t)\right)\left[-\frac{h_{1}+\widetilde{h}_{1}}{2} Q_{0}(t)+\int_{t}^{b} H_{s}^{\prime}(x, t) Q_{0}(x) d x\right] d t=0 .
\end{aligned}
$$


On the base of Riemann-Lebesgue lemma, we have, for sufficiently large $\rho$,

$$
\left\{\begin{array}{l}
\int_{0}^{b} \cos \left(2 \rho t-Q_{+}(t)\right)\left[\frac{1-h_{1} \widetilde{h}_{1}}{2} Q_{1}(t)+\int_{t}^{b} H_{c}^{\prime}(x, t) Q_{1}(x) d x\right] d t=0, \\
\int_{0}^{b} \sin \left(2 \rho t-Q_{+}(t)\right)\left[-\frac{h_{1}+\widetilde{h}_{1}}{2} Q_{1}(t)+\int_{t}^{b} H_{s}^{\prime}(x, t) Q_{1}(x) d x\right] d t=0, \\
\int_{0}^{b} \cos \left(2 \rho t-Q_{+}(t)\right)\left[\frac{1-h_{1} \widetilde{h}_{1}}{2} Q_{0}(t)+\int_{t}^{b} H_{c}^{\prime}(x, t) Q_{0}(x) d x\right] d t=0, \\
\int_{0}^{b} \sin \left(2 \rho t-Q_{+}(t)\right)\left[-\frac{h_{1}+\widetilde{h}_{1}}{2} Q_{0}(t)+\int_{t}^{b} H_{s}^{\prime}(x, t) Q_{0}(x) d x\right] d t=0,
\end{array}\right.
$$

and

$$
\left\{\begin{array}{l}
h_{1}-\widetilde{h}_{1}+2 \int_{0}^{b} Q_{1}(x)\left[\frac{1+h_{1} \widetilde{h}_{1}}{2} \cos Q_{-}(x)+\frac{h_{1}-\widetilde{h}_{1}}{2} \sin Q_{-}(x)\right] d x=0, \\
h_{0}-\widetilde{h}_{0}+\int_{0}^{b} Q_{0}(x)\left[\frac{1+h_{1} \widetilde{h}_{1}}{2} \cos Q_{-}(x)+\frac{h_{1}-\widetilde{h}_{1}}{2} \sin Q_{-}(x)\right] d x=0 .
\end{array}\right.
$$

Equations (3.9) and the completeness of "cos" and " $\sin$ " [5] result in that, for $k=0,1$,

$$
Q_{k}(t)+\int_{t}^{b} H_{c}^{\prime \prime}(x, t) Q_{k}(x) d x=0=Q_{k}(t)+\int_{t}^{b} H_{s}^{\prime \prime}(x, t) Q_{k}(x) d x,
$$

where the functions $H_{c}^{\prime \prime}(x, t)$ and $H_{s}^{\prime \prime}(x, t)$ are bounded. These homogeneous Volterra integral equations have only zero solution $Q_{k}(x)=0, k=0,1$ for $x \in(0, b)$. So, $q_{k}(x)=\widetilde{q}_{k}(x), k=$ 0,1 a.e. on $[0, b]$. Moreover, from (3.10), one can easily get that $h_{k}=\tilde{h_{k}}, k=0,1$.

By use of the change of variable $x \rightarrow \pi-x$, the segment $(b, \pi)$ is converted to the segment $(0, \pi-b)$. So, by repeating the pervious discussions for the supplementary problem $\widehat{L}:=$ $L\left(\mathrm{q}_{1}, \mathrm{q}_{0}, H_{1}, H_{0}, h_{1}, h_{0}, \alpha, \beta, \gamma\right)$, we get

$$
\begin{aligned}
& -y^{\prime \prime}+\left(2 \rho \mathrm{q}_{1}(x)+\mathrm{q}_{0}(x)\right) y=\lambda y, \quad x \in(0, \pi), \\
& \mathrm{q}_{k}(x)=q_{k}(\pi-x), \quad k=0,1, \\
& U(y):=y^{\prime}(0)+\left(H_{1} \rho+H_{0}\right) y(0)=0, \\
& V(y):=y^{\prime}(\pi)-\left(h_{1} \rho+h_{0}\right) y(\pi)=0, \\
& y\left(\frac{\pi}{2}+0, \rho\right)=\alpha^{-1} y\left(\frac{\pi}{2}-0, \rho\right), \\
& y^{\prime}\left(\frac{\pi}{2}+0, \rho\right)=\alpha y^{\prime}\left(\frac{\pi}{2}-0, \rho\right)-(\beta \rho+\gamma) y\left(\frac{\pi}{2}-0, \rho\right),
\end{aligned}
$$

the subject is proved on $(b, \pi)$. Since the conditions of Lemma 3.2 are satisfied to $\widehat{L}$, we can similarly give that $\mathrm{Q}_{k}(x)=Q_{k}(\pi-x)=0, k=0,1$ on $(0, \pi-b)$. So $q_{k}(x)=\widetilde{q}_{k}(x), k=0,1$ a.e. on $[b, \pi]$ and $H_{k}=\widetilde{H}_{k}, k=0,1$. The proof is completed.

Proof of Theorem 3.1 According to Lemma 3.2 and taking the assumptions $\left\langle y_{r(n)}, \tilde{y}_{r(n)}\right\rangle_{x=b}=$ 0 and $\lambda_{n}=\widetilde{\lambda}_{n}$, we imply that $q_{k}(x)=\widetilde{q}_{k}(x)$ on $x \in[b, \pi]$ and $H_{k}=\widetilde{H}_{k}, k=0,1$. Therefore it is enough to show that $q_{k}(x)=\widetilde{q}_{k}(x)$ for $x \in[0, b]$ and $h_{k}=\widetilde{h}_{k}, k=0,1$.

When $b \in\left[\frac{\pi}{2}, \pi\right]$, we have (3.1) as follows:

$$
\mathbb{G}_{b}(\rho):=\int_{0}^{b}\left(2 \rho Q_{1}(x)+Q_{0}(x)\right) y(x) \widetilde{y}(x) d x+\left(h_{1}-\widetilde{h}_{1}\right) \rho+h_{0}-\widetilde{h}_{0}
$$




$$
=\left.\left(y^{\prime}(x) \widetilde{y}(x)-y(x) \widetilde{y}^{\prime}(x)\right)\right|_{\frac{\pi}{2}-0}+\left.\left(y^{\prime}(x) \widetilde{y}(x)-y(x) \widetilde{y}^{\prime}(x)\right)\right|_{\frac{\pi}{2}+0} ^{b} .
$$

Because $y_{n}(x)$ and $\tilde{y}_{n}(x)$ have a similar condition in $x=\pi$ and $\widetilde{q}_{k}(x)=q_{k}(x), k=0,1$ on $x \in[b, \pi]$, we infer that

$$
y_{n}(x)=\alpha_{n} \tilde{y}_{n}(x), \quad n \in \mathbb{N}, x \in[b, \pi]
$$

for constants $\alpha_{n}$. Together with (3.16) and equality $\langle y, z>\rangle_{x=\frac{\pi}{2}-0}=\left.\langle y, z\rangle\right|_{x=\frac{\pi}{2}+0}$, this yields $\mathbb{G}_{b}\left(\lambda_{n}\right)=0$ and analogously $\mathbb{G}_{b}\left(\mu_{l(n)}\right)=0$.

The total of roots $\lambda_{n}$, i.e., $n_{\lambda_{n}}(r)=1+r[1+\epsilon(r)]$, and $\mu_{l(n)}$, i.e., $n_{\mu_{l(n)}}(r)=1+r \sigma_{1}[1+\epsilon(r)]$, inside the disc of radius $r$ is $n(r)=2+r\left[1+\sigma_{1}+\epsilon(r)\right]$. So we get, for $\sigma_{1}>\frac{2 b}{\pi}-1$,

$$
\lim _{r \rightarrow \infty} \frac{n(r)}{r} \geq\left(1+\sigma_{1}\right)>\frac{1}{2 \pi} \int_{0}^{2 \pi} h(\theta) d \theta
$$

Lemma 2.1 together with (3.18) yields that $\mathbb{G}_{b}(\lambda)=0$.

Now, similar to the proof of Lemma 3.2, we can show that $q_{k}(x)=\widetilde{q}_{k}(x)$ a.e. on $[0, b]$ and $h_{k}=\widetilde{h}_{k}, k=0,1$. The proof is completed.

Another result of this paper is achieved by the Gesztesy-Simon method, in which a subset of eigenvalues and partial information on the potentials are used to establish the uniqueness theorem for $L$.

Theorem 3.3 Consider $\sigma(L)$ as the spectrum of $L$. If the coefficient $H_{k}$ and the potentials $q_{k}(x), k=0,1$ are provided on $[\pi / 2(1-\alpha), \pi]$ for some $\alpha \in(0,1)$, then a subset $S \subseteq \sigma(L)$ satisfying the following inequality for sufficiently small $\epsilon>0$ and whole large enough $\lambda_{0} \in \mathbb{R}$

$$
\sharp\left\{\lambda \in S ; \lambda \leq \lambda_{0}\right\} \geq(1-\alpha) \sharp\left\{\lambda \in \sigma(L) ; \lambda \leq \lambda_{0}\right\}+\frac{\alpha}{2}+\epsilon
$$

is sufficient to determine the coefficient $h_{k}$ and the potentials $q_{k}(x), k=0,1$ on $[0, \pi]$.

Proof Multiplying (1.1) by $\widetilde{y}(x, \rho)$ and the corresponding equation by $y(x, \rho)$, using the difference of these results and integrating on $[0, \pi]$, we infer that

$$
\begin{aligned}
& \int_{0}^{\pi}\left(2 \rho Q_{1}(x)+Q_{0}(x)\right) y(x) \widetilde{y}(x) d x \\
& \quad=\left.\left(y^{\prime}(x) \widetilde{y}(x)-y(x) \widetilde{y}^{\prime}(x)\right)\right|_{0} ^{\frac{\pi}{2}-0}+\left.\left(y^{\prime}(x) \widetilde{y}(x)-y(x) \widetilde{y}^{\prime}(x)\right)\right|_{\frac{\pi}{2}+0} ^{\pi} .
\end{aligned}
$$

By regards to $q_{k}(x)=\widetilde{q}_{k}(x), k=0,1$, for $x \in[\pi / 2(1-\alpha), \pi]$, we can give

$$
\begin{aligned}
G_{\alpha}(\rho) & :=\int_{0}^{\pi / 2(1-\alpha)}\left(2 \rho Q_{1}(x)+Q_{0}(x)\right) y(x) \widetilde{y}(x) d x+\left(h_{1}-\widetilde{h}_{1}\right) \rho+h_{0}-\widetilde{h}_{0} \\
& =y^{\prime}(\pi) \widetilde{y}(\pi)-y(\pi) \widetilde{y}^{\prime}(\pi) .
\end{aligned}
$$

On the base of the assumption of the theorem, we have $G_{\alpha}\left(\rho_{n}\right)=0$ for $\rho_{n} \in S$. Now we must show that $G_{\alpha}(\rho)=0$ for all $\rho$. 
By virtue of (3.4) and (3.20), we infer that

$$
G_{\alpha}(\rho) \leq\left(M_{1}^{\prime}+M_{2}^{\prime}|\rho|\right) \exp (|\Im \rho|(1-\alpha) \pi)
$$

for constants $M_{1}^{\prime}, M_{2}^{\prime}>0$. Hence, for $\lambda=i x$, one obtains that

$$
G_{\alpha}(i x) \leq\left(M_{1}^{\prime}+M_{2}^{\prime}|\sqrt{x}|\right) \exp (\Im \sqrt{i}|\sqrt{x}|(1-\alpha) \pi)
$$

We denote an entire function

$$
\phi(\rho)=\frac{G_{\alpha}(\rho)}{\Delta_{\alpha}(\rho)}
$$

where $\Delta_{\alpha}(\rho)=\prod_{\lambda_{n} \in S}\left(1-\frac{\lambda}{\lambda_{n}}\right)$. Inasmuch as the characteristic function $\Delta(\rho)$ is an entire function of order $\frac{1}{2}$, there exists a positive constant $C$ such that

$$
N_{\Delta_{\alpha}}\left(\rho_{0}\right) \leq N_{\Delta}\left(\rho_{0}\right) \leq C \sqrt{\lambda}
$$

in which $N_{\Delta_{\alpha}}\left(\rho_{0}\right):=\sharp\left\{\lambda \in S ; \lambda \leq \lambda_{0}\right\}$ and $N_{\Delta}\left(\rho_{0}\right):=\sharp\left\{\lambda \in \sigma(L) ; \lambda \leq \lambda_{0}\right\}$. Using the hypothesis of the theorem, we get

$$
N_{\Delta_{\alpha}}\left(\rho_{0}\right) \geq(1-\alpha) N_{\Delta}\left(\rho_{0}\right)+\frac{\alpha}{2}+\epsilon
$$

Some standard computations in Refs. $[7,28]$ conclude that

$$
\ln \left|\Delta_{\alpha}(i x)\right|=(1-\alpha) \ln |\Delta(i x)|+\left(\frac{\alpha}{4}+\frac{\epsilon}{2}\right) \ln \left(1+x^{2}\right) .
$$

Consequently,

$$
\left|\Delta_{\alpha}(i x)\right|=|\Delta(i x)|^{1-\alpha} \cdot\left(1+x^{2}\right)^{\frac{\alpha}{4}+\frac{\epsilon}{2}}
$$

Since $\sigma(L)$ is the spectrum of $L$, we have

$$
|\Delta(i x)| \geq C \sqrt{|x|} \exp (\mathfrak{I} \sqrt{i}|\sqrt{x}| \pi)
$$

for sufficiently large $x$. Now, together with (3.24), this gives

$$
\left|\Delta_{\alpha}(i x)\right| \geq C \sqrt{|x|^{(1-\alpha)}} \exp (\Im \sqrt{i}|\sqrt{x}|(1-\alpha) \pi) \cdot\left(1+x^{2}\right)^{\frac{\alpha}{4}+\frac{\epsilon}{2}} .
$$

This result together with (3.21) and (3.22) implies that

$$
\phi(i x)=O\left(\frac{1}{|x|^{\epsilon}}\right)
$$

From Lemma 2.2, we can get $\phi(\rho)=0$ for all $\rho$, and therefore $G_{\alpha}(\rho)=0$ for all $\rho$.

Now, by repeating the arguments in the same manner with Theorem 3.1 , we get $q_{k}(x)=$ $\widetilde{q}_{k}(x)$ a.e. on $[0, \pi / 2(1-\alpha)]$ and $h_{k}=\widetilde{h}_{k}, k=0,1$. The proof is completed. 


\section{Acknowledgements}

The authors would like to thank the anonymous referees for their valuable comments and suggestions which helped to improve the paper.

Funding

Not applicable.

\section{Availability of data and materials}

Not applicable.

\section{Competing interests}

The authors declare that they have no competing interests.

Authors' contributions

All authors contributed equally and significantly in writing this article. All authors read and approved the final manuscript.

\section{Author details}

'Department of Basic Sciences, Sari Agricultural Sciences and Natural Resources University, 578, Sari, Iran. ${ }^{2}$ Department of Mathematics, Cankaya University, Ankara, Turkey.

\section{Publisher's Note}

Springer Nature remains neutral with regard to jurisdictional claims in published maps and institutional affiliations.

Received: 27 August 2020 Accepted: 15 December 2020 Published online: 22 December 2020

\section{References}

1. Ambartsumyan, V.A.: Uber eine frage der eigenwerttheorie. Z. Phys. 53, 690-695 (1929)

2. Anderssen, R.S.: The effect of discontinuities in density and shear velocity on the asymptotic overtone structure of torsional eigenfrequencies of the Earth. Geophys. J. R. Astron. Soc. 50, 303-309 (1997)

3. Baltes, H.P.: Inverse Scattering Problems in Optics. Topics in Current Physics. Springer, Berlin (1980)

4. Buterin, S.A., Shieh, C.T.: Incomplete inverse spectral and nodal problems for differential pencils. Results Math. 62 , 167-179 (2012)

5. Freiling, G., Yurko, V.A.: Inverse Sturm-Liouville Problems and Their Applications. Nova Publ., New York (2001)

6. Fulton, C.T.: Two-point boundary value problems with eigenparameter contained in the boundary conditions. Proc. R. Soc. Edinb., Sect. A, Math. 77(3-4), 293-308 (1977)

7. Gesztesy, F., Simon, B.: Inverse spectral analysis with partial information on the potential, II. The case of discrete spectrum. Trans. Am. Math. Soc. 352, 2765-2787 (2000)

8. Hald, O.: Discontinuous inverse eigenvalue problems. Commun. Pure Appl. Math. 37, 539-577 (1984)

9. Hochstadt, H., Lieberman, B.: An inverse Sturm-Liouville problem with mixed given data. SIAM J. Appl. Math. 34 676-680 (1978)

10. Khalili, Y., Kadkhoda, N., Baleanu, D.: Inverse problems for the impulsive Sturm-Liouville operator with jump conditions. Inverse Probl. Sci. Eng. 27(10), 1442-1450 (2019)

11. Khalili, Y., Yadollahzadeh, M., Moghadam, M.K.: Half inverse problems for the impulsive operator with eigenvalue-dependent boundary conditions. Electron. J. Differ. Equ. 2017, 190 (2017)

12. Koyunbakan, H., Panakhov, E.: Half-inverse problem for diffusion operators on the finite interval. J. Math. Anal. Appl. 326(3), 1024-1030 (2007)

13. Kraft, R.E., Wells, W.R.: Adjointness properties for differential systems with eigenvalue-dependent boundary conditions, with application to flow-duct acoustics. J. Acoust. Soc. Am. 61(4), 913-922 (1977)

14. Krueger, R.J.: Inverse problems for nonabsorbing media with discontinuous material properties. J. Math. Phys. 23 , 396-404 (1982)

15. Levin, B.J.: Distribution of zeros of entire functions. AMS Transl. 5, (1964)

16. Likov, A.V., Mikhailov, Y.A.: The Theory of Heat and Mass Transfer. Qosenergoizdat, Moscow (1963)

17. Litvinenko, O.N., Soshnikov, V.I.: The Theory of Heterogeneous Lines and Their Applications in Radio Engineering. Radio, Moscow (1964)

18. McLaughlin, J.R., Polyakov, P.L.: On the uniqueness of a spherically symmetric speed of sound from transmission eigenvalues. J. Differ. Equ. 107(2), 351-382 (1994)

19. Meschanov, V.P., Feldstein, A.L.: Automatic Design of Directional Couplers. Sviaz, Moscow (1980)

20. Mochizuki, K., Trooshin, I.: Inverse problem for interior spectral data of Sturm-Liouville operator. J. Inverse III-Posed Probl. 9, 425-433 (2001)

21. Mochizuki, K., Trooshin, I.: Inverse problem for interior spectral data of the Dirac operator on a finite interval. Publ. Res Inst. Math. Sci. 38(2), 387-395 (2002)

22. Neamaty, A., Khalili, Y.: Determination of a differential operator with discontinuity from interior spectral data. Inverse Probl. Sci. Eng. 22(6), 1002-1008 (2013)

23. Ozkan, A.S., Keskin, B.: Spectral problems for Sturm-Liouville operator with boundary and jump conditions linearly dependent on the eigenparameter. Inverse Probl. Sci. Eng. 20(6), 799-808 (2012)

24. Parker, R.L., Whaler, K.A.: Numerical methods for establishing solutions to the inverse problem of electromagnetic induction. J. Geophys. Res. 86(10), 9574-9584 (1981)

25. Sat, M., Panakhov, E.S.: A uniqueness theorem for Bessel operator from interior spectral data. Abstr. Appl. Anal. 2013, Article ID 713654 (2013)

26. Tikhonov, A.N., Samarskii, A.A.: Equations of Mathematical Physics. Pergamon, Oxford (1963) 
27. Voitovich, N.N., Katsenelbaum, B.Z., Sivov, A.N.: Generalized Method of Eigen-Vibration in the Theory of Diffraction. Nauka, Moscow (1997)

28. Wang, Y.P.: A uniqueness theorem for Sturm-Liouville operators with eigenparameter dependent boundary conditions. Tamkang J. Math. 43(1), 145-152 (2012)

29. Wang, Y.P.: Inverse problems for Sturm-Liouville operators with interior discontinuities and boundary conditions dependent on the spectral parameter. Math. Methods Appl. Sci. 36, 857-868 (2013)

30. Wang, Y.P.: Uniqueness theorems for Sturm-Liouville operators with boundary conditions polynomially dependent on the eigenparameter from spectral data. Results Math. 63, 1131-1144 (2013)

31. Wang, Y.P., Yang, C.F., Huang, Z.Y.: Half inverse problem for Sturm-Liouville operators with boundary conditions dependent on the spectral parameter. Turk. J. Math. 37, 445-454 (2013)

32. Yang, C.F., Bondarenko, N.P., Xu, X.C.: An inverse problem for the Sturm-Liouville pencil with arbitrary entire functions in the boundary condition. Inverse Probl. Imaging 14(1), 153-169 (2020)

33. Yang, C.F., Guo, Y.X.: Determination of a differential pencil from interior spectral data. J. Math. Anal. Appl. 375, 284-293 (2011)

34. Yang, C.F., Yu, X.J.: Determination of differential pencils with spectral parameter dependent boundary conditions from interior spectral data. Math. Methods Appl. Sci. 37(6), 860-869 (2014)

35. Yang, C.F., Yurko, V.A.: On the determination of differential pencils with nonlocal conditions. J. Inverse III-Posed Probl. 26(5), 577-588 (2018)

36. Yurko, V.A.: Integral transforms connected with discontinuous boundary value problems. Integral Transforms Spec. Funct. 10(2), 141-164 (2000)

37. Yurko, V.A.: The inverse spectral problem for differential operators with nonseparated boundary conditions. J. Math. Anal. Appl. 250(3), 266-289 (2000)

38. Yurko, V.A., Yang, C.F.: Recovering differential operators with nonlocal boundary conditions. Anal. Math. Phys. 6, $315-326(2016)$

\section{Submit your manuscript to a SpringerOpen ${ }^{\circ}$ journal and benefit from:}

- Convenient online submission

- Rigorous peer review

- Open access: articles freely available online

- High visibility within the field

- Retaining the copyright to your article

Submit your next manuscript at $\gg$ springeropen.com 